\title{
EXTASIS: Radio detection of cosmic rays at low frequencies
}

\author{
Daniel García-Fernández ${ }^{1, * * *}$, Didier Charrier ${ }^{1,3}$, Richard Dallier $^{1,3}$, Antony Escudie $^{1}$, Alain Lecacheux $^{2}$, Lilian $_{\text {Martin }}{ }^{1,3}$, \\ and Benoît Revenu ${ }^{1,3}$ \\ ${ }^{1}$ SUBATECH, Institut Mines-Télécom Atlantique - CNRS/IN2P3 - Université de Nantes, Nantes, France \\ ${ }^{2}$ CNRS - Observatoire de Paris-Meudon, France \\ ${ }^{3}$ Unité Scientifique de Nançay, Observatoire de Paris, CNRS, PSL, UO/OSUC, Nançay, France
}

\begin{abstract}
The detection of cosmic rays via the electric field (the so-called radio detection) is nowadays a fully operative technique. With the aim of exploring the low-frequency part of the emission spectrum (below $10 \mathrm{MHz}$ ), the EXTASIS experiment has been conceived. Located within the CODALEMA experiment at the Nançay radio-observatory, EXTASIS measures the low-frequency emission coming from the extensive air showers created by cosmic rays. Being able to calculate the electric field at low frequency is crucial in order to correctly interpret our results. We present some results from the EXTASIS experiment and discuss the calculation of the low-frequency radio signal. We also present a new formula for the electric field of a particle track within two semi-infinite media (air and soil).
\end{abstract}

\section{Introduction}

Radio detection of cosmic rays is a well-established technique [1] that allows the determination of the relevant properties of the primary cosmic ray by measuring the electric field radiated by the extensive air shower (EAS) induced by the primary cosmic ray. Its arrival direction, primary energy and composition can be measured with uncertainties similar to those obtained with a surface array or a fluorescence telescope. Radio detection is also able to measure composition at energies lower than the fluorescence threshold and its low cost can be exploited to gain more statistics in the ultra-high energy region $\left(>10^{19} \mathrm{eV}\right)$. The radio technique may prove useful for the detection of ultra-high energy neutrinos in ice [2].

Most experiments (such as CODALEMA [3], LOFAR [4], AERA [5] or Tunka-Rex [6]) measure the electric field above $20 \mathrm{MHz}$, where the technique is mastered and the atmospheric noise does not pose a problem for detection. However, in the past some experiments $([7,8])$ have detected a low-frequency $(<10 \mathrm{MHz})$ electric signal associated with EAS. Low frequency implies a large wavelength, so the shower is expected to emit more coherently at these frequencies. We also expect a new kind of signal produced when the shower is suddenly decelerated upon arrival at the ground, which we call the sudden death pulse (SDP) [9].

The EXTASIS experiment has been built with the aim of measuring the electric field of an EAS at low frequencies, from 1.7 to $3.7 \mathrm{MHz}$. In this work, we discuss the emission of an EAS at low frequency, introduce a new formula for the electric field that takes into account the air-

\footnotetext{
*We thank the Région Pays de la Loire for its financial support

**e-mail: daniel.garcia-fernandez@ subatech.in2p3.fr
}

soil boundary and present some results from the EXTASIS experiment.

\section{Simulations of the electric field at low frequency}

Standard codes for the calculation of the electric field (SELFAS2, ZHAireS or CoREAS) work in the far field, meaning that they calculate the electric field under the approximation that the measuring antenna is located in the radiation zone, or equivalently, that the wavenumber $k$ times the distance between the emitter and the observer $R$ is much greater than $1: k R=2 \pi R / \lambda \gg 1$. If an important contribution from the tail of the shower is expected (sudden death mechanism), and the antennas are at a distance smaller than $100 \mathrm{~m}$ from the shower core, at $1 \mathrm{MHz}$, $k R=2 \pi \cdot 100 / 300 \sim 2$, so near-field effects can be expected to be important.

\subsection{Direct emission from a particle track at all frequencies. Sudden death pulse}

As a first approximation, we will ignore the effect of the soil in our calculations. When working in the far-field, this is usually sufficient since the effect of the soil can be taken into account with the reception pattern for the antenna. When calculating in the near-field regime, this is not necessarily true but such an approximation seems reasonable at first order.

Let us define a particle track as the trajectory of a particle that begins at rest, and is suddenly accelerated gaining a velocity $\mathbf{v}$ at $t=t_{1}$. Then, it travels at constant speed for a time and stops suddenly at a time $t=t_{2}$. Monte Carlo 


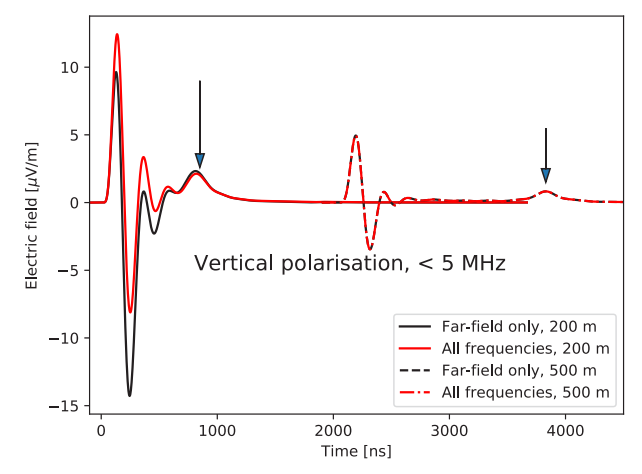

Figure 1. Electric field (vertical component) as a function of time created by a $1 \mathrm{EeV}$ proton-induced shower with $30^{\circ}$ of zenithal angle. Times have been arbitrarily offset. One observer is placed at $200 \mathrm{~m}$ from the shower core and the other one at 500 $\mathrm{m}$. The SDP is indicated by the arrows.

codes for calculating the field from EAS use in fact particle tracks as a way to approximate real particle trajectories. The field for each track is calculated and the total field is obtained as the sum of the field of all the tracks thanks to the superposition principle.

A complete formula for the direct electric field of a track at all frequencies can be found in [9]. This formula is embedded in the MC code SELFAS3, which has been used to elucidate the properties of the low-frequency emission from EAS. In Fig. 1 we show the time trace of the electric field created by a $1 \mathrm{EeV}$ proton shower, filtered with a lowpass filter below $5 \mathrm{MHz}$. There is a difference between the far-field approximation and the exact formula, as well as a bump after the main pulse. This pulse corresponds to the SDP, the emission created by the sudden deceleration of the shower front at ground level. The SDP pulse is received by the antenna at a time directly proportional to the distance between the antenna and the shower core. Moreover, the amplitude of the SDP decreases with the dependence $1 / R$, with $R$ the distance to the shower core. The arrival times and the amplitudes, measured by several antennas, would constitute a new method of direct measurement of shower core position, freed from the use of extensive MC simulations. The SDP pulse disappears at high frequency, since it is created by the coherent deceleration of the shower front, and coherence is most present at large wavelength.

Another feature of the low-frequency field, as predicted by the SELFAS3 code, is that the amplitude of the electric field on the ground does not decrease as fast as with high frequency. Due to the larger wavelength, the increased coherence of the emission creates a much wider profile on the ground. This implies that, if the lowfrequency field is detectable, we would expect it to have a larger detection range compared to the standard [20 - 80] $\mathrm{MHz}$ band.

In Fig. 2 we show the calculated amplitude of the SDP as a function of the shower zenith angle and its energy. For the EXTASIS setup, we do not expect showers above 10

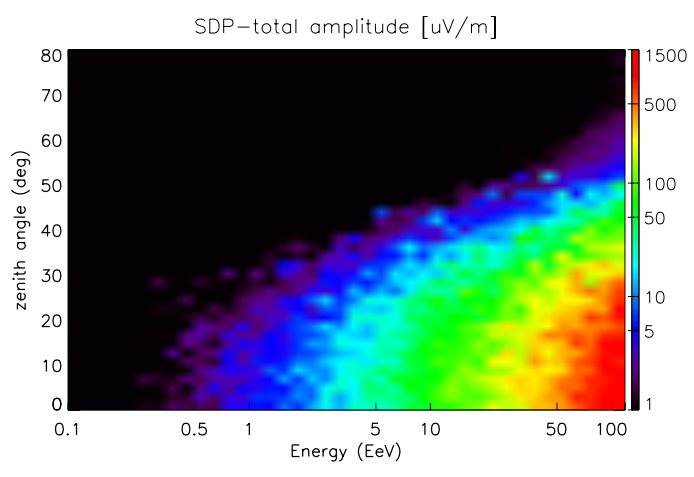

Figure 2. Two-dimensional colour plot of the total SDP maximum amplitude as a function of the primary proton energy and shower zenith angle.

$\mathrm{EeV}$ and we speculate that the detection threshold should be several $\mu \mathrm{V} / \mathrm{m}$, which leaves with a small window for detecting the SDP. At a higher altitude, the SDP emission is enhanced and should be easier to detect.

\subsection{Total emission from a particle track. Surface wave}

The effect of the boundary on the electric field is neglected when working with radiation (far) fields because one of the following approaches suffices. Either we calculate the direct and reflected field and we know the antenna response in free space, or we calculate the direct field and convolve it with the antenna response calculated with the boundary present. Both of these methods should yield the same voltage received by the antenna.

However, when the emitting particle and the antenna are close to the boundary compared to the wavelength, the analysis is not that simple. Near-field effects appear, in particular a type of electric field called surface wave [10]. A way to rigorously calculate the electric field near a boundary is to calculate the field for a dipole following [10]. In particular, we first calculate (in frequency domain) the direct dipole electric field, the electric field from the perfect image of the dipole, and then the surface contribution, that can be known with integrals of the following type:

$$
\int_{0}^{\infty} f\left(\sqrt{k_{1}^{2}-k^{2}}\right) e^{i \sqrt{k_{1}^{2}-k^{2}}} J_{n}(k \rho) k^{3-n} \mathrm{~d} k,
$$

where $k_{1}$ is the wavenumber in the medium where the particle is (air, in our case), $\rho$ is the radial distance to the observer and $f$ is a function that arises from solving Maxwell's equations in Fourier space. $J_{n}$ is a Bessel function of the first kind of order $n$. With these three contributions we can know the field of a perfect dipole, $\mathbf{E}_{\text {dipole }}$, which can be then used to obtain the field from a particle track upon integration:

$$
\mathbf{E}_{\text {track }}(\omega)=q \int_{t_{1}}^{t_{2}} \mathrm{~d} t e^{i \omega t} \mathbf{v} \cdot \mathbf{E}_{\text {dipole }}
$$


We can write as an example the radial field emitted by a vertical track:

$$
\begin{aligned}
& \hat{\rho} \cdot \mathbf{E}_{\text {track }}=\frac{\omega \mu_{0}}{4 \pi k_{1}^{2}} q v \int_{t_{1}}^{t_{2}} \mathrm{~d} t e^{i \omega t} \times \\
& {\left[-e^{i k_{1} r}\left(\frac{i k_{1}^{2}}{r}-\frac{3 k_{1}}{r^{2}}-\frac{3 i}{r^{3}}\right) \frac{\rho}{r} \frac{z-d}{r}\right.} \\
& +e^{i k_{1} r_{\text {image }}}\left(\frac{i k_{1}^{2}}{r_{\text {image }}}-\frac{3 k_{1}}{r_{\text {image }}^{2}}-\frac{3 i}{r_{\text {image }}^{3}}\right) \frac{\rho}{r_{\text {image }}} \frac{z-d}{r_{\text {image }}} \\
& \left.+2 i k_{2}^{2} \int_{0}^{\infty} \frac{\sqrt{k_{1}^{2}-k^{2}} e^{i(z+d)} \sqrt{k_{1}^{2}-k^{2}}}{k_{1}^{2} \sqrt{k_{2}^{2}-k^{2}}+k_{2}^{2} \sqrt{k_{1}^{2}-k^{2}}} J_{1}(k \rho) k^{2} \mathrm{~d} k\right] \text {, }
\end{aligned}
$$

where $k_{2}$ is the wavenumber in the lower medium (soil), $z$ is the height of the antenna, $d$ is the height of the particle track, and $r$ ( $\left.r_{\text {image }}\right)$ is the distance from track (image) to the observer. The second line in Eq. (3) indicates the direct field, while the last two lines indicate the surface wave contribution, expressed as the superposition of the image field and the lateral field. The integral possesses no analytic form or approximation that we are aware of (without making approximations that do not correspond to the EXTASIS experimental setup), and it revealed itself to be quite difficult to compute numerically. In fact, the oscillatory nature of the Bessel functions requires a method for integration known as the Extrapolation Method [11] if we want to reduce the computation time to reasonable levels.

Formulas similar to Eq. (3) exist for the other polarisations and also when the track is underground. We have found that the field underground is always very attenuated for a standard soil, as we expected. We show in Fig. 3 the comparison of the direct, direct plus reflected and exact field for a vertical track $10 \mathrm{~m}$ above ground. We have chosen an atmosphere with a refractive index of $n_{\text {air }}=1.0001$ and a standard soil with $n_{\text {soil }}=13$ and conductivity $\sigma_{\text {soil }}=5 \mathrm{mS} / \mathrm{m}$. We see that the exact field at high frequencies agrees with the direct plus reflected field, as it should be. It is also patent that the surface wave present in the exact calculation amplifies the emission between 1 and $5 \mathrm{MHz}$, the frequency band of EXTASIS. For a track at $100 \mathrm{~m}$ of height, the far-field calculation and the exact calculations match better (Fig. 4). These calculations have yet to be implemented in a Monte Carlo code so as to have a realistic estimation of the boundary effect in air showers.

\section{The EXTASIS experiment}

The EXTASIS experiment has been conceived as an extension to the already existing CODALEMA experiment, located in the Observatory of Nançay, France. CODALEMA [3] is an experiment dedicated to the measurement of cosmic rays, and it is constituted by 13 scintillators acting as a surface detector, 57 self-triggered antennas acting as autonomous stations and 10 antennas forming a compact array. The autonomous stations measure the NorthSouth and East-West components of the electric field in the [20 - 200] MHz band. With the help of this setup, cosmic rays are routinely detected and their arrival direction, energy and composition known with the help of MC simulations.

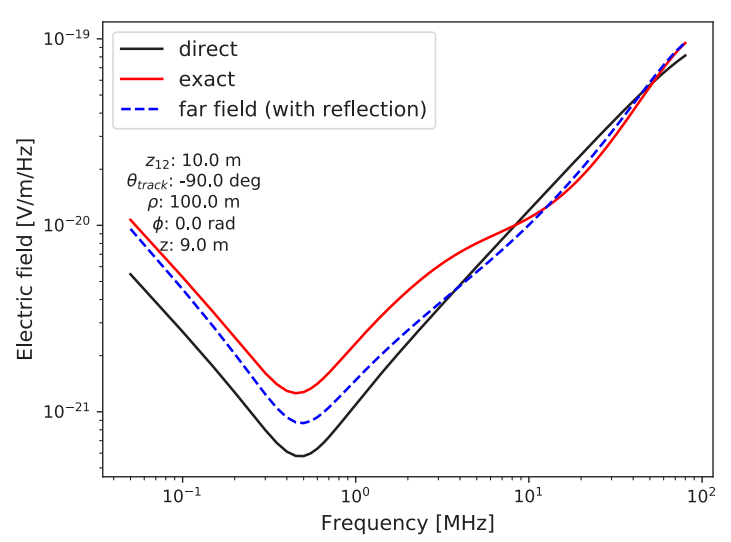

Figure 3. Top: Direct, direct plus reflected (far field) and exact fields for a vertical particle track lying $10 \mathrm{~m}$ above the ground. The observer is located at $9 \mathrm{~m}$ of height at a radial distance of $100 \mathrm{~m}$ from the track.

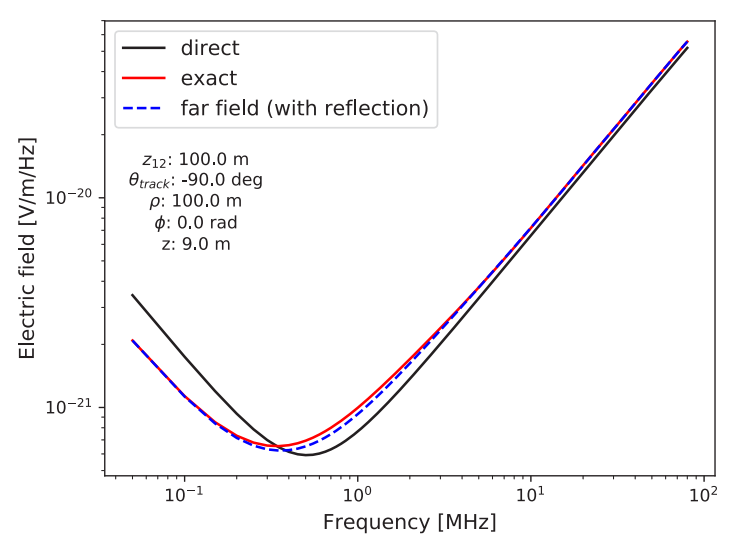

Figure 4. Same as Fig. 4, but with the track at a height of $100 \mathrm{~m}$.

EXTASIS (EXTinction of Air Shower Induced Signal) is the most recent detector in the Nançay observatory. It was designed to detect the low-frequency electric field coming from EAS and, in particular, the SDP emitted when the shower front hits the ground. EXTASIS operates in the $[1-10] \mathrm{MHz}$ band, which is flooded with anthropogenic and atmospheric noise, which restricts the data taking to daytime only, since the noise is highly dependent on the ionosphere temperature.

The current EXTASIS configuration consists of 7 antennas located $9 \mathrm{~m}$ above ground. These antennas measure the vertical and the East-West component of the electric field in the $[1-10] \mathrm{MHz}$ band. They are triggered by the scintillator array of CODALEMA.

So far, EXTASIS has detected several low-frequency events, all of them detected in the $[20$ - 200] $\mathrm{MHz}$ band by CODALEMA. We show one these events in Fig. 5, plotted on a map of the CODALEMA/EXTASIS setup with information on the electric field measured by the antenna array. The arrival directions $((\theta, \phi))$ re- 


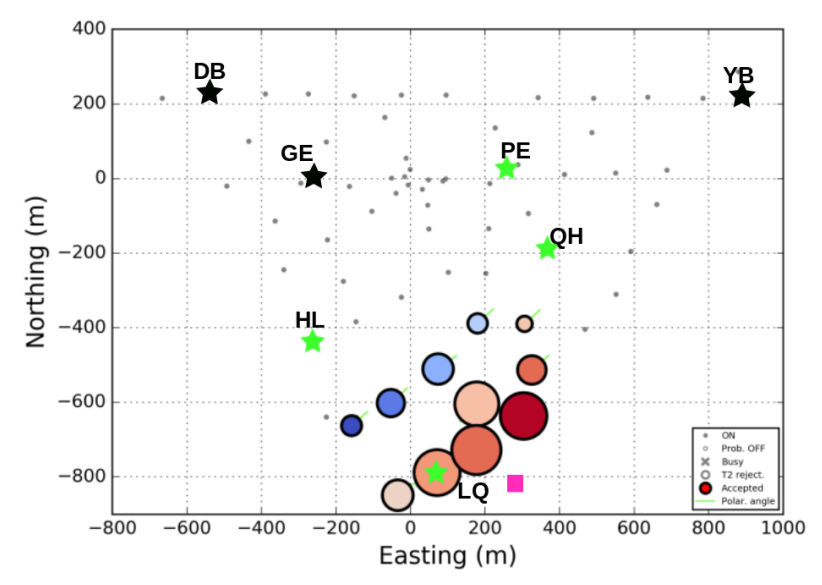

Figure 5. Map indicating the electric field of an event measured by the CODALEMA antennas and the EXTASIS antennas. Coloured circles and grey dots represent the CODALEMA antennas, while the stars depict the low-frequency antennas. The size of the circles indicate the amplitude of the signal and the colour indicates the arrival time of the signal. A black star indicates no low-frequency signal, and a green star indicates the measurement of a low-frequency transient.

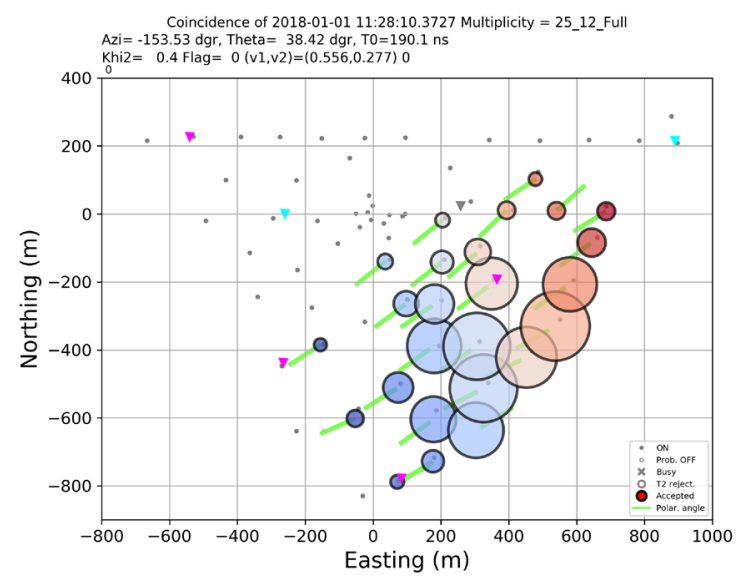

Figure 6. Map indicating the electric field of an event during a thunderstorm measured by the standalone antennas. The green lines indicate the polarisation of the signal. The arrival direction of the shower is almost parallel to the polarisation, which is not expected with the standard geomagnetic mechanism.

constructed by the scintillators $\left(32.4^{\circ}, 144.1^{\circ}\right)$, the autonomous stations $\left(40.6^{\circ}, 145.2^{\circ}\right)$, and the low-frequency array $\left(31.1^{\circ}, 146.1^{\circ}\right)$ are compatible with each other. We see in Fig. 5 that the EXTASIS antennas have detected the signal farther away when compared to the standalone antennas. This hints that the detection range may be indeed larger at low frequencies.

On the downside, we have only had $\sim 15$ lowfrequency events since April 2017, meaning that the ef- ficiency is quite low. Besides, no sudden death signal has been observed so far.

Some of the detected events are correlated with large values of the atmospheric electric field (stormy weather). It is possible that the atmospheric electric field accelerates the particles in the shower similarly to the geomagnetic effect, a process that can be called the geoelectric effect. This effect has been seen by LOFAR [12], and it changes the polarisation of the electric field on the ground inducing an homogeneous polarisation.We show in Fig. 6 an event measured during the Carmen storm, on the 1st of January, 2018. The green arrows that indicate the polarisation reveal that the pattern is quite uniform, and is incompatible with the standard geomagnetic polarisation.

\section{Outlook and conclusions}

EXTASIS is a experiment that measures the low-frequency $([1-10] \mathrm{MHz})$ electric field from EAS. In this work we have studied the expected properties of such an emission, including the SDP. We have introduced a new way of calculating the electric field taking into account the influence of the ground, valid at all frequencies. We have presented some events measured by EXTASIS, that are the low-frequency counterpart of standard radio events. One of this events shows that the detection range seems to be larger at low frequencies. Another of this events shows that the atmospheric electric field can have an important influence on the emission at low frequencies. However, the low number of events detected would suggest that the EXTASIS band is not as exploitable as the standard [30-80] $\mathrm{MHz}$ band. The SDP has not been detected so far.

\section{References}

[1] F.G. Schröder, Prog. Part. Nucl. Phys. 93, 1-68 (2017)

[2] S.W. Barwick et al., Astropart. Phys. 70, 12-26 (2015)

[3] L. Martin et al., Proc.35th ICRC (2017)

[4] P. Schellart et al., Astron. Astrophys. 66, 31 (2015)

[5] The Pierre Auger Coll., Phys. Rev. D 93122005 (2016)

[6] W.D. Apel et al., Phys. Lett. B 763, 179 (2016)

[7] K. Nishi, K. Suga, Proc. 20th ICRC 6, 125 (1987)

[8] C. Castagnoli et al., Proc. 22nd ICRC 4, 363 (1991)

[9] D. García-Fernández et al., Phys. Rev. D 97, 103010 (2018)

[10] R.W.P. King, M. Owens, T.T. Wu, Lateral Electromagnetic Waves (Springer-Verlag, New York, 1992)

[11] K.A. Michalski, IEEE Trans. Antennas Propag. 46, 10 (1998)

[12] P. Schellart et al., Phys. Rev. Lett. 114, 165001 (2015) 